\title{
Smart UV-Spectrophotometric methods for the simultaneous determination of amprolium-HCl, ethopabate and sulfaquinoxaline-Na in combined dosage forms
}

\author{
Shimaa E. Abdelaziz1*, Sawsan A. Abdel Razeq ${ }^{1}$, Nermin S. Ahmed ${ }^{2}$ \\ ${ }^{1}$ Department of Pharmaceutical Analytical Chemistry, Faculty of Pharmacy (Girls), Al-Azhar University, Cairo, Egypt. \\ ${ }^{2}$ Department of Pharmaceutical Chemistry, Faculty of Pharmacy and Biotechnology, German University in Cairo, Cairo \\ 11835, Egypt. \\ * Corresponding author: E-mail: shimaaebrahim.pharmg@azhar.edu.eg
}

\author{
Article history: Received 2021-02-07 Revised 2021-04-16
}

Accepted 2021-04-27

\begin{abstract}
Two UV-spectrophotometric methods were adopted for simultaneous determination of amprolium $\mathrm{HCl}$, ethopabate and sulfaquinoxaline-Na. The two methods are a double divisor ratio derivative method and ratio dual wavelength method. The three cited drugs were determined by the suggested methods in the range of 6.0-50.0 $\mu \mathrm{g} \mathrm{mL}^{-1}, 2.0-27.0 \mu \mathrm{g} \mathrm{mL}^{-1}$ and 3.0-25.0 $\mu \mathrm{g} \mathrm{mL}^{-1}$, respectively. The proposed methods were proved to be selective where the mean percentage recoveries of amprolium HCL, ethopabate and sulfaquinoxaline-Na provided by the double divisor ratio derivative method were 99.29 $\pm 0.84,100.36 \pm 1.21$ and 100.61-101.57 \pm 0.15-1.75; respectively. The ratio dual wavelength method mean recoveries were in the range of 98.93$100.45 \% \pm 0.44-1.42,99.47-100.67 \% \pm 0.83-1.65$ and $99.97-101.71 \% \pm 0.10-1.15$ for the studied drugs; respectively. Both methods were validated following ICH guidelines. Successful application of the methods for analyzing the three drugs in their veterinary dosage forms; Ampoethoquinone powder was conducted. The obtained results were statistically analyzed and found to be in accordance with those given by the reported methods.
\end{abstract}

Keywords: Amprolium; Ethopabate; Sulfaquinoxaline; Ratio Derivative; Ratio Dual Wavelength.

\section{INTRODUCTION}

One of the most common parasitic diseases is coccidiosis. It is caused by coccidian protozoa which infect the intestinal tract of livestock animals. Symptoms include being gaunt, pallor, unwillingness to eat, and diarrhea ${ }^{1}$. A combination of the three drugs; amprolium, ethopabate and sulfaquinoxaline$\mathrm{Na}$; in a single dosage form provides a potent prevention and treatment of coccidiosis.

Amprolium; 1-[(4-amino-2-pro-pyl-5pyrimidinyl) methyl]-2-methylpyridiniumchloride Hydrochloride salt ${ }^{2}$. It is mainly utilized to prevent and treat intestinal coccidiosis through disruption of cell metabolism ${ }^{3}$. Ethopabate; methyl 4(acetylamino)-2-ethoxybenzoat ${ }^{2}$. The drug is a

competitor of PABA absorption by the parasite and interferes with folate synthesis. So, it has been used only in combination with amprolium to provide potent peak activity on the $4^{\text {th }}$ day of parasite cycle ${ }^{4}$. Sulfaquinoxaline-Na, N-4-Amino-N-quinoxalin-2- ylbenzenesulfonamide sodium salt ${ }^{2}$ is a member of sulfonamide antimicrobials. It interferes with the biosynthesis of folic acid so when combined with vitamin $\mathrm{K}$ provides potential treatment of coccidiosis 5 .

Various techniques were conducted for the determination of either amprolium $\mathrm{HCl}$, ethopabate and sulfaquinoxaline- $\mathrm{Na}$ in binary mixtures and/or with other drugs. Among the stated methods; HPLC ${ }^{6-12}$, LC/MS 13-15, TLC-Densitometry 16-18, Fluorimetry 19 UV-Spectrophotometry 18,20 and potentiometry ${ }^{21,22}$. It is noteworthy to mention that there were no analytical methods had been established for the simultaneous determination of amprolium $\mathrm{HCl}$, ethopabate and sulfaquinoxaline- $\mathrm{Na}$ as ternary mixture.

The actual purpose of this study is to achieve successful analysis of amprolium $\mathrm{HCl}$, ethopabate and sulfaquinoxaline- $\mathrm{Na}$ in ternary mixture either in their pure and dosage forms by simple UVspectrophotometric methods.

Cite this article: Abdelaziz, S., Abdel Razeq, S., Ahmed, N. Smart UV-Spectrophotometric methods for the simultaneous determination of amprolium-HCl, ethopabate and sulfaquinoxaline-Na in combined dosage forms. Azhar International Journal of Pharmaceutical and Medical Sciences, 2021; 1(2):63-71. doi: 10.21608/aijpms.2021.62019.1046 
<smiles>CCCc1ncc(C[n+]2ccccc2C)c(N)n1</smiles>

Amprolium HCl

Molecular formula: $\mathrm{C}_{14} \mathrm{H}_{19} \mathrm{ClN}_{4}, \mathrm{HCl}$<smiles>Nc1ccc(S(=O)(=O)N([AlH2])c2cnc3ccccc3n2)cc1</smiles>

Sulfaquinoxaline-Na

Molecular formula: $\mathrm{C}_{14} \mathrm{H}_{12} \mathrm{~N}_{4} \mathrm{O}_{2} \mathrm{~S}$,<smiles>CCOC(=O)c1ccc(NC(C)=O)cc1OCC</smiles>

Ethopabate

Molecular formula: $\mathrm{C}_{14} \mathrm{H}_{15} \mathrm{NO}$.

\section{METHODS}

\subsection{Instruments}

UV-Vis Spectrophotometer (Shimadzu 1601, Japan); Shimadzu UV- Probe version 2.32.

Ultrasonic (Wised clean, China).

\subsection{Materials and Reagent}

Pure Amprolium HCl; B.N. WS/20180717, was kindly supplied by Zhejiang K-sheng., Biopharmgroup Co. LTD, Egypt; with purity of $99.8 \%$ purity as referred by the supplier and it was tested by TLC-densitometry.

Pure Ethopabate; B. N. 20190327, was kindly supplied by Zhejiang huangyan vet Pharma factory, china, with purity of $99.5 \%$ as referred by the supplier and it was tested by TLC-densitometry.

Pure Sulfaquinoxaline-Na; B. N. BL160725, was kindly supplied by wujiang Bolin industry co. Ltd., Egypt; with purity of $99.5 \%$ as referred by the supplier and it was tested by TLC-densitometry.

Amproethoquine powder; B.N. ATQN5097, labeled to contain amprolium $\mathrm{HCl} 200 \mathrm{gm}$, ethopabate 10 gm sulfaquinoxaline-Na 128.78 gm per $1 \mathrm{Kg}$, the product of Biovet, Cairo, Egypt.

Methanol, (Sigma Aldrich, Germany).

\subsection{Standard solutions}

Stock solutions $\left(1.0 \mathrm{mg} \mathrm{mL}^{-1}\right)$ of amprolium $\mathrm{HCl}$, ethopabate or sulfaquinoxaline-Na were prepared by weighing $100.0 \mathrm{mg}$ of each pure drug to be completely dissolved in $100-\mathrm{mL}$ methanol. Then, working solutions $\left(0.1 \mathrm{mg} \mathrm{mL}^{-1}\right)$ were prepared by the suitable dilution using methanol as diluent.

\subsection{Procedures}

\subsubsection{Linearity}

Aliquots from amprolium $\mathrm{HCl}$, ethopabate or sulfaquinoxaline-Na standard solutions in methanol mg or $0.03-0.25 \mathrm{mg}$, respectively; were dissolved in $10 \mathrm{~mL}$ methanol separately. The prepared solutions were scanned over the range of 200.0-400.0 nm and the zero-order spectra were saved in the computer.

\subsubsection{Double divisor ratio spectra derivative method (DDRD):}

The zero-order spectra of one drug were divided by the sum of the absorption spectra of the other two drugs "double divisor". The resultant ratio spectra were derivatized using with $\Delta \lambda=8.0 \mathrm{~nm}$ and scaling factor $=100$. Amprolium $\mathrm{HCl}$ and ethopabate were detected at 238.6 and $233.0 \mathrm{~nm}$; respectively. While sulfaquinoxaline-Na amplitudes were at 234.0, 242.0, 263.0 and $289.0 \mathrm{~nm}$. The amplitudes of each drug were recorded and plotted against amprolium $\mathrm{HCl}$, sulfaquinoxaline- $\mathrm{Na}$ and ethopabate concentration; respectively.

\subsubsection{Ratio dual wavelength method (RDW):}

Employing amprolium $\mathrm{HCl}$ as a devisor: The zero-order spectra of each of ethopabate and sulfaquinoxaline-Na were divided by amprolium $\mathrm{HCl}$ spectrum $\left(20.0 \mu \mathrm{g} \mathrm{mL} \mathrm{mL}^{-1}\right)$. Each drug would be estimated at the amplitude difference at one or more wavelengths couples where a reasonable linearity and accepted recovery was obtained. Ethopabate was determined at $(214.0-229.0 \mathrm{~nm}),(244.0-262.0 \mathrm{~nm})$ and (254.0-248.0 nm). While sulfaquinoxaline-Na was assessed at (224.0-247.0 $\mathrm{nm})$ and (229.0-245.0 $\mathrm{nm})$.

Using ethopabate as a devisor: Similarly, the spectra of each of amprolium $\mathrm{HCl}$ and sulfaquinoxaline-Na were divided by $20.0 \mu \mathrm{g} \mathrm{mL}^{-1}$ ethopabate spectrum. Amprolium $\mathrm{HCl}$ was measured at (236.0- $247.0 \mathrm{~nm})$ and (258.0-283.0 $\mathrm{nm}$ ). Likewise, sulfaquinoxaline-Na could be determined at (228.0-246.0 nm), (233.0-241.0 nm) and (255.0$271.0 \mathrm{~nm})$.

Applying sulfaquinoxaline-Na as a devisor: Upon dividing amprolium $\mathrm{HCl}$ or ethopabate spectra 
by sulfaquinoxaline-Na spectrum $\left(20.0 \mu \mathrm{g} \mathrm{mL}^{-1}\right)$, the wavelengths couples were $(279.0-319.0 \mathrm{~nm}),(285.0$ $320.0 \mathrm{~nm})$ and $(296.0-316.0 \mathrm{~nm})$ for amprolium $\mathrm{HCl}$, while $(214.0-229.0 \mathrm{~nm}),(255.0-299.0 \mathrm{~nm}),(257.0$ $289.0 \mathrm{~nm})$ and (261-294 $\mathrm{nm})$ for ethopabate.

\subsubsection{Assay of laboratory prepared mixtures of the two drugs}

Different aliquots of amprolium $\mathrm{HCl}$, ethopabate and sulfaquinoxaline-Na working solutions equivalent to $0.06-0.50 \mathrm{mg}, 0.02-0.27 \mathrm{mg}$ or 0.03 $0.25 \mathrm{mg}$, respectively; were transferred into a set of $10-\mathrm{mL}$ volumetric flaks. Then, methanol is used as diluent. The spectra of the obtained solutions were scanned. The drugs concentrations were calculated using their corresponding regression equations just after following the manipulating steps for each method as described under "2.4.1. Linearity".

\subsubsection{Applications to dosage forms}

Accurately weighed $0.5 \mathrm{gm}$ of Amproethoquine - powder equivalent to $100.0 \mathrm{mg}$ amprolium $\mathrm{HCl}, 5.0$ $\mathrm{mg}$ ethopabate and $64.0 \mathrm{gm}$ sulfaquinoxaline-Na was transferred into $100-\mathrm{mL}$ volumetric flask. Volume was completed with methanol to prepare a solution claimed to contain $1.00 \mathrm{mg} \mathrm{mL}^{-1}$ amprolium $\mathrm{HCl}$, $0.05 \mathrm{mg} \mathrm{mL} \mathrm{mL}^{-1}$ ethopabate and $0.64 \mathrm{mg}$ sulfaquinoxaline-Na. Further dilutions within the linearity range were prepared and analyzed following the procedures detailed under "2.4.1. Linearity" and "2.4.2. Assay of laboratory prepared mixtures of the three drugs". The drugs concentrations were calculated from the corresponding regression equations.

\section{RESULTS AND DISCUSSION}

The measured absorption spectra of amprolium $\mathrm{HCl}$, ethopabate and sulfaquinoxaline- $\mathrm{Na}$ showed sever overlapping; Figure 1. A problem that restricts their simultaneous estimation of the three drugs either in mixtures or in dosage forms. Although the drugs were analyzed using a wide diversity of many UV spectrophotometric methods, there is no published articles describing simultaneous determination of the three studied drugs.

\subsection{Double divisor ratio spectra derivative method (DDRD)}

The principle of the DDRD method applied the derivative technique to the ratio spectra. Moreover, ratio spectrum of one drug is calculated by dividing the spectrum of the ternary mixture by a standard spectrum representing the sum of the other two compounds in the title mixture. Also, the quantitative measurements could be at either the maximum or minimum wavelengths ${ }^{23,24}$.

Various spectrophotometric parameters were investigated to obtain maximum resolution and sensitivity. Firstly, the concentration of the standard binary mixture used as double divisor should be determined precisely. Different concentrations were tried where $20 \mu \mathrm{g} \mathrm{mL}^{-1}$ of each drug was the most appropriate concentration to be used as a devisor regarding signal-to-noise ratio.

Moreover, the influence of each of the derivative order, $\Delta \lambda$ and the scaling factor were studied. Although both first and second derivative were tried but ${ }^{3} \mathrm{D}$ had achieved the most acceptable results regarding the obtained recovery. Best results were obtained upon applying $\Delta \lambda=8.0$ and scaling factor $=100$ for the third derivative spectra.

The measured spectra of amprolium $\mathrm{HCl}$ was divided by the sum of the spectra of each of sulfaquinoxaline-Na and ethopabate $\left(20.0 \mu \mathrm{g} \mathrm{mL}^{-1}\right)$ as a "double divisor" to get ratio spectra. ${ }^{3} \mathrm{D}$ of the ratio spectra was calculated applying $\Delta \lambda=8.0$ and scaling factor $=100$ where amprolium $\mathrm{HCl}$ was determined at 229.0 and $238.6 \mathrm{~nm}$; Figure 2. The same procedure was followed for ethopabate estimation using amprolium $\mathrm{HCl}$ and sulfaquinoxaline-Na as a "double divisor"; Figure 3. While amprolium $\mathrm{HCl}$ and ethopabate were employed as a "double divisor" for sulfaquinoxaline-Na determination; Figure 4. There was a reasonable linearity at 233.0, 250.0 and $315.0 \mathrm{~nm}$ for ethopabate. While sulfaquinoxaline-Na was determined at 234.0, 242.0, 263.0 and $289.0 \mathrm{~nm}$.

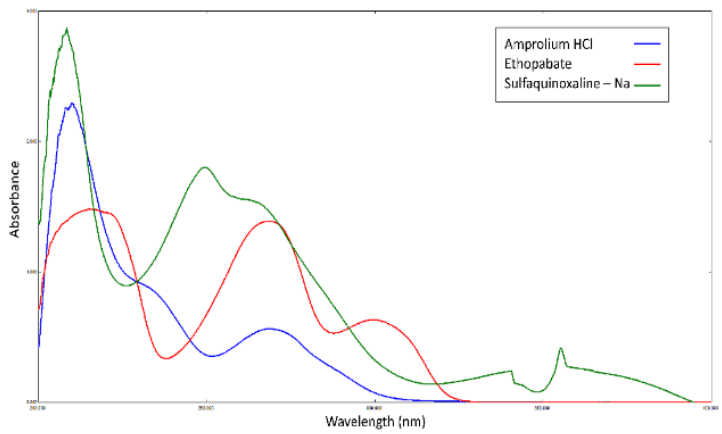

Figure 1: Zero-order absorption spectra of $20 \mu \mathrm{g} \mathrm{mL}^{-1}$ of each of amprolium $\mathrm{HCl}$, ethopabate and sulfaquinoxaline$\mathrm{Na}$ in methanol.

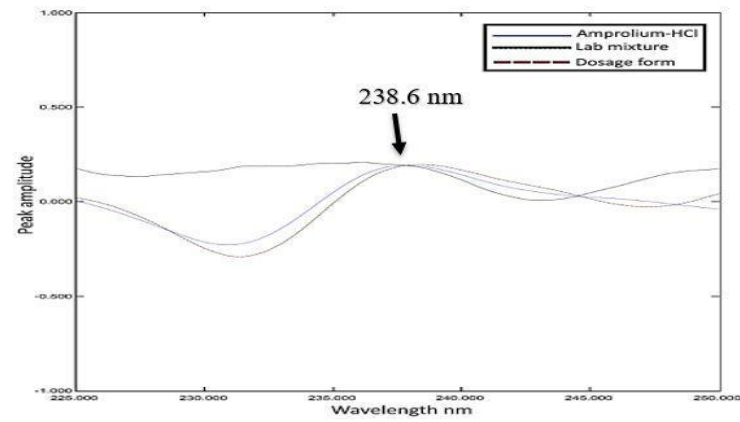

Figure 2: Third derivative of ratio spectra of amprolium $\mathrm{HCl}\left(20.0 \mu \mathrm{g} \mathrm{mL}^{-1}\right)$ in pure form, dosage form and 
laboratory prepared mixture using sulfaquinoxaline-Na (20.0 $\left.\mu \mathrm{g} \mathrm{mL}^{-1}\right)$ and ethopabate $\left(20.0 \mu \mathrm{g} \mathrm{mL}^{-1}\right)$ as a double divisor.

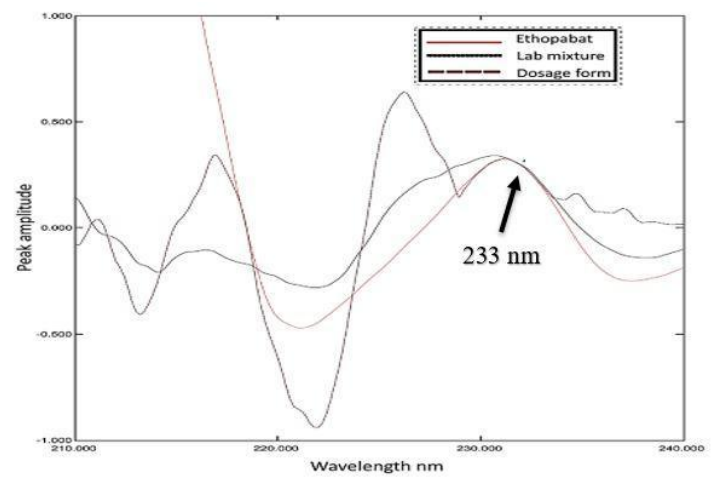

Figure 3: Third derivative of ratio spectra of ethopabate $\left(20.0 \mu \mathrm{g} \mathrm{mL}^{-1}\right)$ in pure form, dosage form and laboratory prepared mixture using amprolium $\mathrm{HCl}\left(20.0 \mu \mathrm{g} \mathrm{mL}^{-1}\right)$ and sulfaquinoxaline- $\mathrm{Na}\left(20.0 \mu \mathrm{g} \mathrm{mL}^{-1}\right)$ as a double divisor.

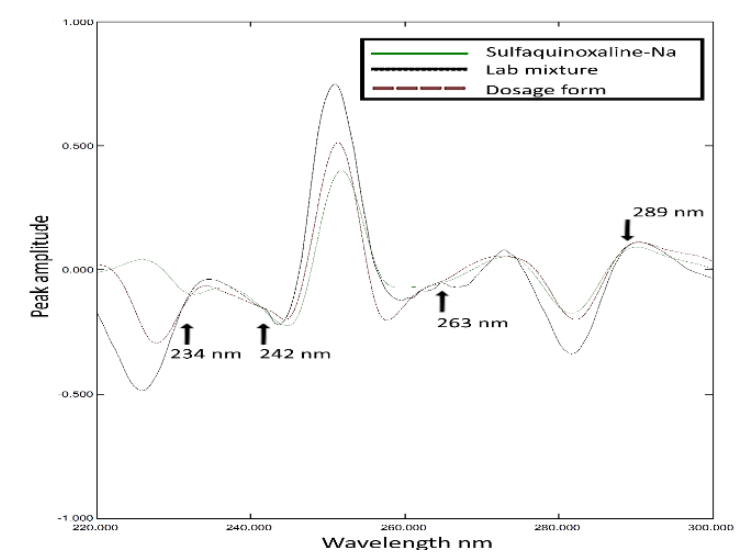

Figure 4: Third derivative of ratio spectra of sulfaquinoxaline- $\mathrm{Na}\left(12.0 \mu \mathrm{g} \mathrm{mL}^{-1}\right)$ in pure form, dosage form and laboratory prepared mixture using amprolium $\mathrm{HCl}$ $\left(20.0 \mu \mathrm{g} \mathrm{mL}^{-1}\right)$ and ethopabate $\left(20.0 \mu \mathrm{g} \mathrm{mL}^{-1}\right)$ as a double divisor.

\subsection{Ratio dual wavelength method (RDW)}

Simply, the principles of both ratio difference method and dual wavelength method were combined so that the resulted method had proven its merits in determining compounds selectively in ternary mixtures. Each component could be selectively determined after complete removal of the interference exerted by the other two components using one component as a divisor and choosing two wavelengths showing equal absorptivity's for the second component 25. Firstly, the devisor concentration should be selected to obtain ratio spectra. Then, one wavelength couple at least showing equal absorptivities was specified for quantitative determination of each drug.

Different divisor concentrations were studied where $20.0 \mu \mathrm{g} \mathrm{mL}^{-1}$ of each drug was suitable based on maximum selectivity and minimal noise. For the proposed ternary mixture of amprolium $\mathrm{HCl}$, ethopabate and sulfaquinoxaline- $\mathrm{Na}$, each drug was estimated at the amplitude difference at one or more wavelengths couples. The amplitude differences between different wavelength couples were tried, where couples only showed reliable results regarding selectivity and recovery were selected.

The ratio spectra of the three drugs were obtained after using a divisor of $20.0 \mu \mathrm{g} \mathrm{mL} \mathrm{m}^{-1}$ of ethopabate. Ethopabate appears as a straight line with constant amplitude, whereas the sulfaquinoxaline-Na shows equal amplitudes at (236 and $247 \mathrm{~nm}$ ) and (258 and $283 \mathrm{~nm}$ ); Figure 5. Therefore, the difference in amplitudes between these two wavelengths would be selectively corresponding to amprolium $\mathrm{HCl}$ concentration. Amprolium $\mathrm{HCl}$ could be also determined between (279 and $319 \mathrm{~nm}),(285$ and 320 $\mathrm{nm}$ ) and (296 and $316 \mathrm{~nm}$ ) using $20.0 \mu \mathrm{g} \mathrm{mL}-1$ of sulfaquinoxaline- $\mathrm{Na}$ as divisor at which ethopabate showed equal amplitudes and sulfaquinoxaline-Na appears straight line with constant amplitude; Figure 6.

The determination of ethopabate was conducted after dividing the spectra of the three drugs by the spectrum of $20.0 \mu \mathrm{g} \mathrm{mL}^{-1}$ of sulfaquinoxaline-Na; Figure 6. So that sulfaquinoxaline-Na was showed as a straight line with constant amplitude, whereas the amprolium $\mathrm{HCl}$ had equal amplitudes at (214.0 and $229.0 \mathrm{~nm}),(255.0$ and $299.0 \mathrm{~nm}),(257.0$ and 289.0 $\mathrm{nm})$ and $(216.0$ and $294.0 \mathrm{~nm})$ at which ethopabate was selectively determined. Moreover, ethopabate could be determined at $(214.0$ and $229.0 \mathrm{~nm}),(242.0$ and $262.0 \mathrm{~nm})$ and $(248.0$ and $254.0 \mathrm{~nm})$ using amprolium $\mathrm{HCl}\left(20.0 \mu \mathrm{g} \mathrm{mL}^{-1}\right)$ as divisor at which sulfaquinoxaline-Na exhibited equal amplitudes; Figure 7.

Similarly, RDW was applied for sulfaquinoxaline-Na determination using ethopabate $\left(20.0 \mu \mathrm{g} \mathrm{mL}^{-1}\right) \quad$ or amprolium $\mathrm{HCl}(20.0 \mu \mathrm{g}$ $\left.\mathrm{mL}^{-1}\right)$ as a divisor; Figure 7. The selected wavelengths couples are $(224.0$ and $247.0 \mathrm{~nm})$ and $(229.0$ and $245.0 \mathrm{~nm})$ applying amprolium $\mathrm{HCl}\left(20.0 \mu \mathrm{g} \mathrm{mL}^{-1}\right)$ as a divisor, while $(228.0$ and $246.0 \mathrm{~nm}),(233.0$ and $241.0 \mathrm{~nm})$ and (255.0 and $271.0 \mathrm{~nm}$ ) using ethopabate $\left(20.0 \mu \mathrm{g} \mathrm{mL}^{-1}\right)$ as a divisor; Figure 5 .

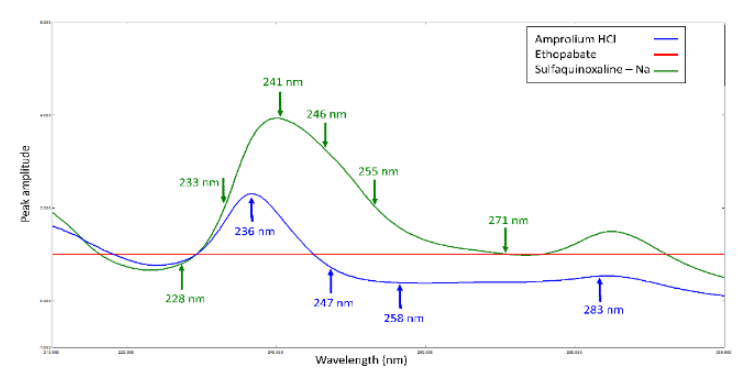


Figure 5: Ratio spectra of $20.0 \mu \mathrm{g} \mathrm{mL} L^{-1}$ of each of amprolium $\mathrm{HCl}$, ethopabate and sulfaquinoxaline-Na using $20.0 \mu \mathrm{g} \mathrm{mL}^{-1}$ ethopabate as a divisor.

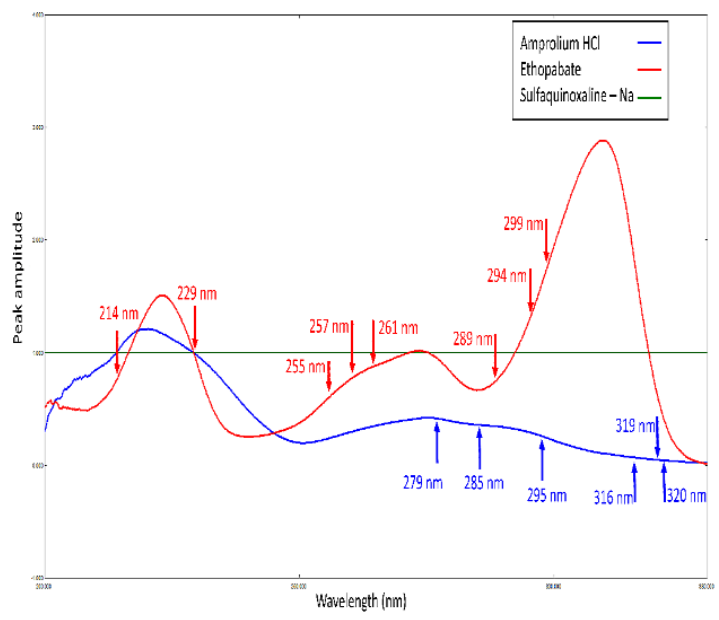

Figure 6: Ratio spectra of $20.0 \mu \mathrm{g} \mathrm{mL}^{-1}$ of each of amprolium $\mathrm{HCl}$, ethopabate and sulfaquinoxaline-Na using $20.0 \mu \mathrm{g} \mathrm{mL}^{-1}$ sulfaquinoxaline-Na as a divisor.

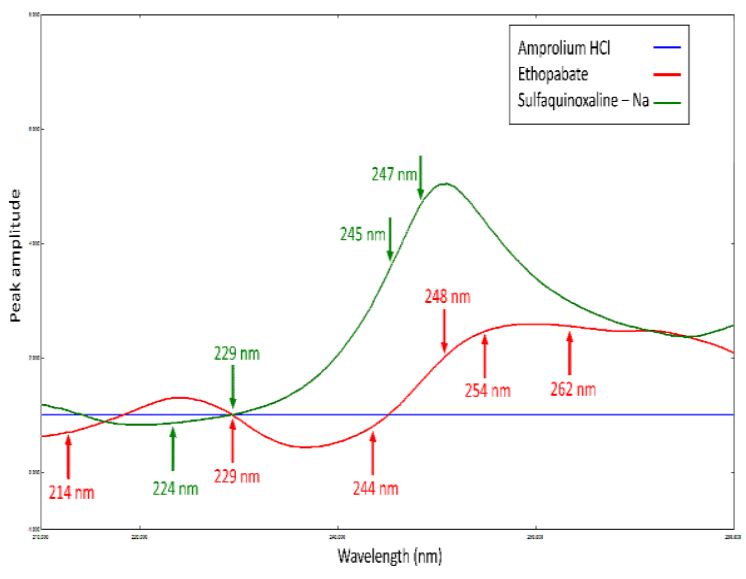

Figure 7: Ratio spectra of $20.0 \mu \mathrm{g} \mathrm{mL}^{-1}$ of each of amprolium $\mathrm{HCl}$, ethopabate and sulfaquinoxaline-Na using $20.0 \mu \mathrm{g} \mathrm{mL}^{-1}$ amprolium $\mathrm{HCl}$ as a divisor.

\subsection{Method validation}

The methods were validated according to the ICH guidelines ${ }^{26}$.

\subsubsection{Linearity}

Good linear relationship was found between the peak amplitude and the corresponding drug concentration over the range of $6.0-50.0 \mu \mathrm{g} \mathrm{mL}^{-1}, 2.0$ - $27.0 \mu \mathrm{g} \mathrm{mL}^{-1}$ and 3.0 - $25.0 \mu \mathrm{g} \mathrm{mL}^{-1}$ for amprolium $\mathrm{HCl}$, ethopabate and sulfaquinoxaline-Na, respectively by proposed UV- spectrophotometric methods; Table 1, 2.

\subsubsection{Accuracy}

It was checked by analysis of three triplicate determinations of different concentrations within the linearity range of amprolium $\mathrm{HCl}(10.0,18.0,35.0 \mu \mathrm{g}$
$\left.\mathrm{mL}^{-1}\right)$, ethopabate $\left(4.0,7.0,11.0 \mu \mathrm{g} \mathrm{mL} \mathrm{m}^{-1}\right)$ and sulfaquinoxaline- $\mathrm{Na}\left(6.0,12.0,20.0 \mu \mathrm{g} \mathrm{mL}^{-1}\right)$ in the same day and within three successive days. Accuracy range was found to be $99.76-100.32 \% \pm 0.16-1.63$, $99.45-100.60 \% \pm 0.71-1.32$ and 98.63-100.84 \pm 0.29 1.08 for the three drugs, respectively; Table 1, 2 .

\subsubsection{Precision}

The repeatability and reproducibility of the proposed UV-spectrophotometric methods were confirmed by calculating intraday and interday RSD $\%$. The results ranged from $0.11-1.99 \%, 0.04 \%-$ 1.84 and $0.23 \%-1.95 \%$ for the three drugs, respectively; Table $1,2$.

\subsubsection{Selectivity}

It was revealed by analyzing laboratory prepared mixtures of amprolium $\mathrm{HCl}$, ethopabate and sulfaquinoxaline-Na in different ratios. Simultaneous determination of the three drugs without any interference was achieved. Thus, the established methods were proved to be selective as indicated by the obtained recoveries; Table 3.

Amprolium $\mathrm{HCl}$ was determined in the presence of ethopabate and sulfaquinoxaline- $\mathrm{Na}$ with mean recovery\% of 99.29 by ${ }^{3}$ DDRR method, and 99.68 , $98.93,100.45,100.12$ and 99.74 by RDW method.

While the percentage recovery sulfaquinoxaline-Na estimation was 101.67, 100.96, 101.15 and 100.61 by ${ }^{3}$ DDRR and $100.34,101.71$, $100.60,100.45$ and 99.97 by RDW method.

Furthermore, for ethopabate assessment, ${ }^{3}$ DDRR showed recoveries \pm SD of $100.36 \% \pm 1.21$, while RDW recoveries \pm SD were found to be $99.47 \%$ $\pm 0.83,99.67 \% \pm 0.83,99.56 \% \pm 0.92,100.67 \% \pm$ $1.28,100.20 \% \pm 1.65,99.32 \% \pm 1.12,100.05 \% \pm$ 1.14 .

The selectivity of the proposed method was further assessed by analyzing of the studied drugs in their veterinary dosage form; Amproethoquine powder. The obtained results had confirmed that the proposed spectrophotometric methods were valid for the simultaneous determination of the three drugs in their combined dosage forms without any interference from each other and from excipients and additives; Table 4.

The validity of the proposed methods was further checked by applying the standard addition technique; Table 4. The obtained results were statistically 27 compared with those obtained from the reported methods. ${ }^{1} \mathrm{D}$ UV-spectrophotometry was stated for the simultaneous determination of amprolium $\mathrm{HCl}$ and ethopabate at $239.0 \mathrm{~nm}$ and 246.5 $\mathrm{nm}$; respectively ${ }^{20}$. While the reported method for sulfaquinoxaline-Na was a TLC-densitometry using chloroform: methanol $(9: 1, \mathrm{v} / \mathrm{v})$ as a mobile phase ${ }^{18}$. 
Table 4 Showed that calculated t- and F- values were less than the theoretical ones with respect to accuracy and precision within a probability of $95 \%$, assuring the absence of the significance difference between the proposed and the reported methods. Moreover, the proposed methods were proved to be more selective applying less sophisticated apparatus.

\subsubsection{Stability of standard solutions}

The stability of the standard solutions of amprolium $\mathrm{HCl}$, sulfaquinoxaline-Na and ethopabate $\left(0.1 \mathrm{mg} \mathrm{mL}^{-1}\right)$ in methanol were evaluated by the UVspectrophotometric methods. The solutions were found to be stable for more than one month at room temperature or in refrigerator.

Table 1: Regression parameters for the determination of amprolium $\mathrm{HCl}$ and ethopabate by the proposed UVSpectrophotometric methods

\begin{tabular}{|c|c|c|c|c|c|c|c|c|c|c|c|c|c|c|}
\hline \multirow{2}{*}{\begin{tabular}{|l} 
Parameters \\
$\lambda_{\max }(n m)$
\end{tabular}} & \multicolumn{2}{|c|}{$\begin{array}{c}\text { Using ethopabate as a } \\
\text { divisor }\end{array}$} & \multicolumn{3}{|c|}{$\begin{array}{l}\text { using sulfaquinoxaline-Na as a } \\
\text { divisor }\end{array}$} & \multirow{2}{*}{$\begin{array}{c}{ }^{3} D D R D \\
238.6\end{array}$} & \multicolumn{4}{|c|}{ using sulfaquinoxaline-Na as a divisor } & \multicolumn{3}{|c|}{$\begin{array}{c}\text { using amprolium HCL as a } \\
\text { divisor }\end{array}$} & \multirow{2}{*}{$\begin{array}{r}{ }^{3} D D R D \\
233.0\end{array}$} \\
\hline & $\begin{array}{l}236.0 \text { and } \\
247.0\end{array}$ & $\begin{array}{c}283.0 \& \\
258.0\end{array}$ & $\begin{array}{c}296.0 \& \\
316.0\end{array}$ & $\begin{array}{c}285.0 \& \\
320.0\end{array}$ & $\begin{array}{c}279.0 \& \\
319.0\end{array}$ & & $\begin{array}{l}255.0 \& \\
299.0\end{array}$ & $\begin{array}{c}294.0 \& \\
261.0\end{array}$ & $\begin{array}{c}214.0 \& \\
229.0\end{array}$ & $\begin{array}{c}257.0 \& \\
289.0\end{array}$ & $\begin{array}{l}214.0 \\
\& 229.0\end{array}$ & $\begin{array}{l}244.0 \\
\& 262.0\end{array}$ & $\begin{array}{l}248.0 \\
\& 254.0\end{array}$ & \\
\hline Linearity range & \multicolumn{6}{|c|}{$6.0-50.0 \mu \mathrm{g} \mathrm{mL} L^{-1}$} & \multicolumn{8}{|c|}{$2.0-27.0 \mu \mathrm{g} \mathrm{mL^{-1 }}$} \\
\hline Slope \pm S.D. & $\begin{array}{l}0.07839 \pm \\
1.5 \mathrm{E} 10^{-4}\end{array}$ & $\begin{array}{c}0.00704 \pm \\
2.2 \mathrm{E}^{-5}\end{array}$ & $\begin{array}{c}0.00951 \pm \\
3.6 \mathrm{E}^{-5}\end{array}$ & $\begin{array}{l}0.0145 \pm \\
2.29 \mathrm{E}^{-5}\end{array}$ & $\begin{array}{c}0.01749 \pm \\
5.72 \mathrm{E}^{-5}\end{array}$ & $\begin{array}{l}0.011 \pm \\
3.89 \mathrm{E}^{-5}\end{array}$ & $\begin{array}{c}0.06058 \pm \\
1.6 \mathrm{E}^{-4}\end{array}$ & $\begin{array}{c}0.01777 \pm \\
4.13 \mathrm{E}^{-5}\end{array}$ & $\begin{array}{c}0.01421 \pm \\
4.15 \mathrm{E}^{-5}\end{array}$ & $\begin{array}{c}0.005826 \pm \\
1.19 \mathrm{E}^{-5}\end{array}$ & $\begin{array}{c}0.01348 \pm \\
2.65 \mathrm{E}-{ }^{5}\end{array}$ & $\begin{array}{l}0.08363 \pm \\
2.7 \mathrm{E}^{-4}\end{array}$ & $\begin{array}{c}0.04287 \pm \\
1.4 \mathrm{E}^{-4}\end{array}$ & $\begin{array}{l}0.008 \pm \\
2.44 \mathrm{E}^{-5}\end{array}$ \\
\hline Intercept \pm S.D. & $\begin{array}{r}0.0307 \pm \\
0.004319\end{array}$ & $\begin{array}{c}0.0786 \pm \\
6.4 \mathrm{E}^{-4}\end{array}$ & $\begin{array}{c}0.0214 \pm \\
8.7 \mathrm{E}^{-4}\end{array}$ & $\begin{array}{c}0.0238 \pm \\
6.5 \mathrm{E}^{-4}\end{array}$ & $\begin{array}{l}0.003616 \\
\pm 1.6 \mathrm{E}^{-3}\end{array}$ & $\begin{array}{c}-0.082 \pm \\
1.112 \mathrm{E}^{-3}\end{array}$ & $\begin{array}{c}0.03120 \pm \\
3.08 \mathrm{E}^{-3}\end{array}$ & $\begin{array}{l}0.020836 \\
\pm 7.6 \mathrm{E}^{-4}\end{array}$ & $\begin{array}{c}0.0100 \pm \\
7.7 \mathrm{E}^{-4}\end{array}$ & $\begin{array}{c}0.001217 \pm \\
2.2 \mathrm{E}^{-4}\end{array}$ & $\begin{array}{c}0.01044 \pm \\
6.8 \mathrm{E}^{-4}\end{array}$ & $\begin{array}{c}0.02666 \pm \\
5.1 \mathrm{E}^{-3}\end{array}$ & $\begin{array}{c}0.026015 \\
\pm 2.6 \mathrm{E}^{-3}\end{array}$ & $\begin{array}{l}0.009 \pm \\
4.55 \mathrm{E}^{-4}\end{array}$ \\
\hline S.D. of residual & $4.84 \mathrm{E}^{-3}$ & $8.70 \mathrm{E}^{-4}$ & $1.19 \mathrm{E}^{-3}$ & $8.86 \mathrm{E}^{-4}$ & $2.22 \mathrm{E}^{-3}$ & $1.51 \mathrm{E}^{-3}$ & $3.51 \mathrm{E}^{-3}$ & $8.75 \mathrm{E}^{-4}$ & $8.80 \mathrm{E}^{-4}$ & $2.53 \mathrm{E}^{-4}$ & $7.73 \mathrm{E}^{-4}$ & $5.89 \mathrm{E}^{-3}$ & $3.00 \mathrm{E}^{-3}$ & $4.17 \mathrm{E}^{-4}$ \\
\hline $\begin{array}{l}\text { Coefficient of } \\
\text { determinations }\end{array}$ & 0.9999 & 0.9999 & 0.9999 & 0.9999 & 0.9999 & 0.9999 & 0.9999 & 0.9999 & 0.9999 & 0.9999 & 0.9999 & 0.9999 & 0.9999 & 0.9999 \\
\hline $\begin{array}{l}\text { Accuracy } \\
(R \% \pm \text { S.D. })^{*}\end{array}$ & $\begin{array}{l}101.20 \\
\pm 1.10\end{array}$ & $\begin{array}{l}100.25 \\
\pm 0.16\end{array}$ & $\begin{array}{r}100.16 \\
\pm 1.63\end{array}$ & $\begin{array}{l}99.76 \\
\pm 0.89\end{array}$ & $\begin{array}{l}100.32 \\
\pm 1.45\end{array}$ & $\begin{array}{c}100.28 \\
\pm 0.63\end{array}$ & $\begin{array}{l}100.60 \\
\pm 0.67\end{array}$ & $\begin{array}{l}99.45 \\
\pm 0.48\end{array}$ & $\begin{array}{l}99.45 \\
\pm 1.32\end{array}$ & $\begin{array}{l}100.42 \\
\pm 1.06\end{array}$ & $\begin{array}{l}100.12 \\
\pm 0.96\end{array}$ & $\begin{array}{c}100.12 \\
\pm 0.71\end{array}$ & $\begin{array}{c}100.50 \\
\pm 0.91\end{array}$ & $\begin{array}{c}100.38 \\
\pm 0.98\end{array}$ \\
\hline
\end{tabular}

*mean of nine determinations., Ratio dual wavelength method (RDW)

Table 2: Regression parameters for the determination of sulfaquinoxaline-Na by the proposed UVSpectrophotometric methods

\begin{tabular}{|c|c|c|c|c|c|c|c|c|c|}
\hline \multirow{4}{*}{\begin{tabular}{|l} 
Parameters \\
$\lambda_{\max }(n m)$ \\
\end{tabular}} & \multicolumn{9}{|c|}{ Sulfaquinoxaline } \\
\hline & \multicolumn{5}{|c|}{$R D W$} & \multirow{2}{*}{\multicolumn{4}{|c|}{${ }^{3} D D R D$}} \\
\hline & \multicolumn{3}{|c|}{ using ethopabate as a divisor } & \multicolumn{2}{|c|}{ using amprolium $\mathrm{HCl}$ as a divisor } & & & & \\
\hline & $\begin{array}{c}228.0 \& \\
246.0\end{array}$ & $\begin{array}{l}233 \& \\
241.0\end{array}$ & $\begin{array}{c}255.0 \& \\
271.0\end{array}$ & $\begin{array}{c}224.0 \& \\
247.0\end{array}$ & $\begin{array}{c}229.0 \& \\
245.0\end{array}$ & 234.0 & 242.0 & 263.0 & 289.0 \\
\hline Linearity rang & \multicolumn{9}{|c|}{$3.0-25.0 \mu \mathrm{g} \mathrm{mL^{-1 }}$} \\
\hline Slope \pm S.D. & $\begin{array}{l}0.1467 \pm \\
1.65 \mathrm{E}^{-4}\end{array}$ & $\begin{array}{l}0.106 \pm \\
3.04 \mathrm{E}^{-4}\end{array}$ & $\begin{array}{l}0.0407 \pm \\
1.23 \mathrm{E}^{-4}\end{array}$ & $\begin{array}{l}0.1827 \pm \\
5.51 \mathrm{E}^{-4}\end{array}$ & $\begin{array}{l}0.1381 \pm \\
2.26 \mathrm{E}^{-4}\end{array}$ & $0.006 \pm 1.51 \mathrm{E}^{-5}$ & $0.014 \pm 4.85 \mathrm{E}^{-5}$ & $0.004 \pm 1.45 \mathrm{E}^{-5}$ & $0.0102 \pm 2.31 \mathrm{E}^{-5}$ \\
\hline Intercept \pm S.D. & $\begin{array}{l}-0.3245 \pm \\
2.832 \mathrm{E}^{-3}\end{array}$ & $\begin{array}{c}0.02329 \pm \\
5.24 \mathrm{E}^{-3}\end{array}$ & $\begin{array}{l}-0.01251 \pm \\
2117 \mathrm{E}^{-3}\end{array}$ & $\begin{array}{c}0.1491 \pm \\
9.4964 \mathrm{E}^{-3}\end{array}$ & $\begin{array}{l}0.8549 \pm \\
3.873 \mathrm{E}^{-3}\end{array}$ & $0.0149 \pm 2.59 \mathrm{E}^{-4}$ & $0.01439 \pm 8.33 E^{-4}$ & $0.011 \pm 2.48 \mathrm{E}^{-4}$ & $-0.011 \pm 5.5 \mathrm{E}^{-4}$ \\
\hline S.D. of residual & $2.977 \mathrm{E}^{-3}$ & $5.509 \mathrm{E}^{-3}$ & $2.226 \mathrm{E}^{-3}$ & $9.95 \mathrm{E}^{-3}$ & $4.072 \mathrm{E}^{-3}$ & $2.72 \mathrm{E}^{-4}$ & $8.65 \mathrm{E}^{-4}$ & $2.71 \mathrm{E}^{-4}$ & $5.78 \mathrm{E}^{-4}$ \\
\hline $\begin{array}{l}\text { Coefficient of } \\
\text { determinations }\end{array}$ & 0.9999 & 0.9999 & 0.9999 & 0.9999 & 0.9999 & 0.9999 & 0.9999 & 0.9999 & 0.9999 \\
\hline $\begin{array}{l}\text { Accuracy } \\
(R \% \pm \text { S.D. })^{*}\end{array}$ & $99.57 \pm 1.08$ & $98.63 \pm 0.42$ & $100.51 \pm 0.38$ & $100.84 \pm 0.79$ & $99.85 \pm 0.29$ & $99.94 \pm 0.40$ & $100.38 \pm 1.21$ & $99.55 \pm 0.49$ & $99.81 \pm 0.56$ \\
\hline
\end{tabular}

*mean of nine determinations., Double divisor ratio spectra derivative method (DDRD), Ratio dual wavelength method (RDW). 
Table 3: Determination of amprolium $\mathrm{HCl}$, ethopabate and sulfaquinoxaline-Na in their laboratory prepared mixtures by the proposed UV-Spectrophotometric methods

\begin{tabular}{|c|c|c|c|c|c|c|c|c|c|c|c|c|c|c|c|c|c|c|c|c|c|c|c|c|c|}
\hline \multicolumn{3}{|c|}{$\begin{array}{c}\text { Drugs conc. } \\
\left(\mu g \mathrm{~mL}^{-1}\right)\end{array}$} & \multicolumn{17}{|c|}{ RDW method } & \multicolumn{6}{|c|}{${ }^{3}$ DDRD method } \\
\hline \multirow{2}{*}{ 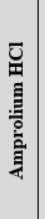 } & \multirow{2}{*}{ 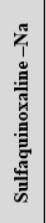 } & \multirow{2}{*}{ 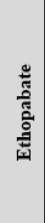 } & 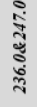 & 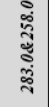 & 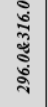 & 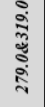 & 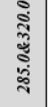 & 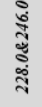 & 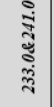 & 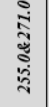 & $\begin{array}{l}\text { वें } \\
\text { वेँ } \\
\text { ปें }\end{array}$ & 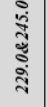 & 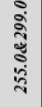 & 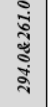 & 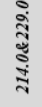 & 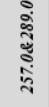 & 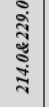 & 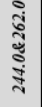 & 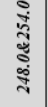 & 邑 & 苛 & 発 & 总 & 品 & ঐ. \\
\hline & & & \multicolumn{5}{|c|}{$\begin{array}{c}\text { Recovery \% of } \\
\text { Amprolium HCl }\end{array}$} & \multicolumn{5}{|c|}{$\begin{array}{c}\text { Recovery \% of } \\
\text { Sulfaquinoxaline }-\mathbf{N a}\end{array}$} & \multicolumn{7}{|c|}{$\begin{array}{l}\text { Recovery \% of } \\
\text { Ethopabate }\end{array}$} & $\begin{array}{c}\text { Recovery \% of } \\
\text { Amprolium } \\
\text { HCl }\end{array}$ & \multicolumn{4}{|c|}{$\begin{array}{c}\text { Recovery \% of } \\
\text { Sulfaquinoxaline }-\mathrm{Na}\end{array}$} & $\begin{array}{c}\text { Recovery } \\
\text { \% of } \\
\text { Ethopabate }\end{array}$ \\
\hline $20^{*}$ & $12^{*}$ & $\mathbf{1}^{*}$ & के & $\frac{m}{\sigma}$ & $\begin{array}{l}\stackrel{a}{\oplus} \\
\stackrel{\square}{\Xi}\end{array}$ & $\begin{array}{l}\text { E } \\
\stackrel{8}{0}\end{array}$ & $\begin{array}{l}\vec{\Xi} \\
\stackrel{\Xi}{\Xi}\end{array}$ & 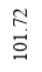 & $\begin{array}{l}\infty \\
\vdots \\
\vdots \\
\vdots\end{array}$ & $\begin{array}{l}\stackrel{8}{\circ} \\
\stackrel{9}{0}\end{array}$ & 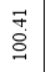 & 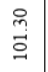 & $\begin{array}{l}\overrightarrow{1} \\
\stackrel{\Xi}{\Xi}\end{array}$ & $\begin{array}{l}\infty \\
\stackrel{\infty}{0} \\
\stackrel{0}{0}\end{array}$ & 官 & 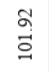 & $\stackrel{m}{\infty}$ & 궁 & $\begin{array}{l}\vec{F} \\
\stackrel{\leftrightarrow}{\circ}\end{array}$ & $\begin{array}{l}n \\
\vdots \\
\alpha \\
\alpha\end{array}$ & $\begin{array}{l}\overrightarrow{+} \\
\stackrel{2}{\circ}\end{array}$ & $\begin{array}{l}\infty \\
\stackrel{\infty}{\Xi} \\
\stackrel{a}{0}\end{array}$ & $\begin{array}{l}0 \\
\vdots \\
0\end{array}$ & \begin{tabular}{|l|}
$n$ \\
$\hat{o}$ \\
0 \\
0
\end{tabular} & $\begin{array}{l}\text { +े } \\
\text { : }\end{array}$ \\
\hline 20 & 20 & 20 & 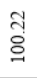 & $\begin{array}{l}f \\
\stackrel{f}{\infty} \\
\infty\end{array}$ & $\begin{array}{l}\circ \\
\stackrel{9}{\Xi}\end{array}$ & $\begin{array}{l}\text { I. } \\
\text { g }\end{array}$ & ळీ & $\begin{array}{l}\text { ?. } \\
\stackrel{9}{\circ}\end{array}$ & $\begin{array}{l}0 \\
\dot{0} \\
0\end{array}$ & $\begin{array}{l}\tilde{I} \\
\stackrel{\Xi}{\Xi}\end{array}$ & 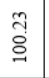 & $\begin{array}{l}\tilde{n} \\
\infty \\
\infty\end{array}$ & $\begin{array}{l}\text { t⿱艹 } \\
2 \\
2\end{array}$ & $\underset{\infty}{m}$ & 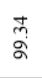 & $\begin{array}{l}\infty \\
\stackrel{\infty}{\circ} \\
\text { ळ }\end{array}$ & $\begin{array}{l}\infty \\
\stackrel{0}{0} \\
\end{array}$ & 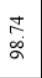 & $\begin{array}{l}\infty \\
\vdots \\
\vdots \\
2\end{array}$ & 웅 & $\begin{array}{l}\overrightarrow{ \pm} \\
\stackrel{0}{0} \\
\stackrel{0}{0}\end{array}$ & $\begin{array}{l}\overrightarrow{7} \\
\vec{\Xi}\end{array}$ & $\begin{array}{l}\infty \\
\stackrel{0}{\Xi}\end{array}$ & \begin{tabular}{|l|}
$a$ \\
$\dot{a}$ \\
0 \\
0
\end{tabular} & $\begin{array}{l}\dddot{a} \\
\stackrel{-}{\Xi}\end{array}$ \\
\hline 10 & 20 & 20 & ồ & ò & 恕 & $\begin{array}{l}0 \\
+ \\
\stackrel{0}{+}\end{array}$ & $\begin{array}{l}\text { I } \\
\text { S }\end{array}$ & สั & $\begin{array}{l}\hat{n} \\
\vdots \\
0\end{array}$ & $\stackrel{\circ}{\circ}$ & $\begin{array}{l}\vec{n} \\
\overrightarrow{0}\end{array}$ & mo & $\hat{\hat{o}}$ & సે & 年 & \% & $\begin{array}{l}\hat{0} \\
\infty \\
\infty\end{array}$ & $\underset{\infty}{\infty}$ & $\underset{\infty}{7}$ & 㕝 & 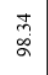 & $\begin{array}{l}\text { in } \\
\text { s. }\end{array}$ & $\stackrel{m}{\vec{\Xi}}$ & $\begin{array}{l}1 \\
2 \\
2 \\
2\end{array}$ & $\stackrel{\infty}{2}$ \\
\hline 20 & 10 & 20 & $\begin{array}{l}\vec{a} \\
\text { ळo }\end{array}$ & $\begin{array}{l}f \\
\text { fo } \\
\text { o }\end{array}$ & 옹 & ๙ิ & $\begin{array}{l}0 \\
\stackrel{0}{0} \\
\stackrel{0}{0}\end{array}$ & $\begin{array}{l}\text { ôे } \\
\stackrel{0}{0}\end{array}$ & $\underset{D}{\stackrel{i}{a}}$ & $\begin{array}{l}\overrightarrow{0} \\
\dot{0}\end{array}$ & $\begin{array}{l}\text { t. } \\
\stackrel{0}{0}\end{array}$ & $\tilde{2}$ & $\underset{\substack{\circ \\
a}}{\stackrel{0}{a}}$ & $\begin{array}{l}\overrightarrow{0} \\
\text { a }\end{array}$ & $\begin{array}{l}\stackrel{0}{0} \\
\text { ă }\end{array}$ & $\begin{array}{l}\text { : } \\
\stackrel{0}{0}\end{array}$ & 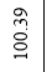 & 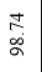 & $\begin{array}{l}\text { N } \\
\stackrel{0}{\circ}\end{array}$ & @ & 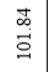 & $\begin{array}{l}\text { fo } \\
\stackrel{0}{0}\end{array}$ & $\begin{array}{l}\text { సे } \\
\stackrel{-}{0}\end{array}$ & $\begin{array}{l}0 \\
0 \\
0 \\
0\end{array}$ & $\underset{\substack{n \\
\infty}}{n}$ \\
\hline 20 & 20 & 10 & $\begin{array}{l}7 \\
\stackrel{5}{\circ}\end{array}$ & aे & ลิ & $\begin{array}{l}\overrightarrow{7} \\
\text { aे }\end{array}$ & $\begin{array}{l}\text { ț } \\
\stackrel{2}{\circ}\end{array}$ & $\begin{array}{l}\text { qे } \\
\text { gे }\end{array}$ & $\begin{array}{l}\infty \\
\stackrel{\infty}{0} \\
\stackrel{a}{a}\end{array}$ & $\begin{array}{l}\tilde{2} \\
\text { S̆ }\end{array}$ & $\begin{array}{l}\text { ?े } \\
\text { gे }\end{array}$ & $\begin{array}{l}\hat{0} \\
\stackrel{0}{0}\end{array}$ & $\begin{array}{l}\infty \\
\infty \\
\infty\end{array}$ & $\begin{array}{l}\infty \\
\grave{0} \\
\stackrel{0}{0}\end{array}$ & ले & $\stackrel{\circ}{\circ}$ & $\begin{array}{l}\stackrel{2}{े} \\
\stackrel{a}{0}\end{array}$ & $\begin{array}{l}\vec{m} \\
\vec{\Xi}\end{array}$ & $\begin{array}{l}\stackrel{\circ}{ } \\
\stackrel{8}{0}\end{array}$ & ڤ̆ & $\begin{array}{l}\hat{0} \\
\overrightarrow{0}\end{array}$ & $\begin{array}{l}\stackrel{q}{f} \\
\vec{g}\end{array}$ & $\begin{array}{l}\stackrel{n}{n} \\
\stackrel{0}{0}\end{array}$ & $\begin{array}{l}\vec{b} \\
\dot{d} \\
\dot{a}\end{array}$ & $\begin{array}{l}\stackrel{\infty}{\oplus} \\
\stackrel{\sim}{\vec{D}}\end{array}$ \\
\hline & $40 \%$ & & 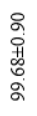 & 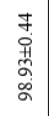 & 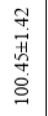 & 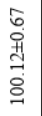 & $\begin{array}{c}\vec{a} \\
\text { ò } \\
\text { 贲 } \\
\text { à }\end{array}$ & 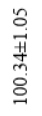 & $\begin{array}{l}\stackrel{0}{0} \\
\stackrel{0}{+} \\
\stackrel{+}{0} \\
\stackrel{0}{0}\end{array}$ & 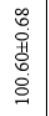 & 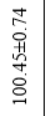 & $\begin{array}{l}\stackrel{\sim}{2} \\
\vec{H} \\
\sigma \\
\alpha \\
\alpha\end{array}$ & 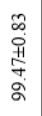 & 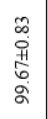 & 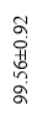 & $\begin{array}{l}\text { व } \\
\overrightarrow{\#} \\
6 \\
0 \\
0\end{array}$ & 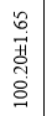 & 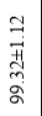 & 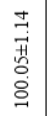 & 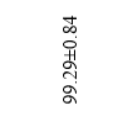 & $\begin{array}{l}n \\
\stackrel{2}{H} \\
\vec{H} \\
6 \\
\stackrel{0}{0}\end{array}$ & 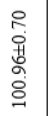 & 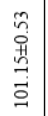 & $\mid \begin{array}{l}\overrightarrow{0} \\
\overrightarrow{0} \\
\vec{H} \\
\overrightarrow{0} \\
\dot{0} \\
0\end{array}$ & $\begin{array}{l}\vec{T} \\
\text { H艹 } \\
\tilde{O} \\
\stackrel{0}{0}\end{array}$ \\
\hline
\end{tabular}

*mean of nine determinations., Double divisor ratio spectra derivative method (DDRD), Ratio dual wavelength method (RDW).

Table 4: Statistical analysis of the result obtained by the proposed UV and reported methods for the determination amprolium $\mathrm{HC}$, ethopabate and sulfaquinoxaline-Na in their pure form

\begin{tabular}{|c|c|c|c|c|c|c|c|c|c|c|c|c|c|c|c|c|c|c|c|c|c|c|c|c|c|c|}
\hline \multirow[b]{4}{*}{ Parameters } & \multicolumn{7}{|c|}{ Amprolium HCl } & \multicolumn{9}{|c|}{ Ethopabate } & \multicolumn{10}{|c|}{ Sulfaquinoxaline $-\mathrm{Na}$} \\
\hline & \multicolumn{6}{|c|}{ UV-Spectrophotometric methods } & \begin{tabular}{|l} 
Reported \\
method $^{* *}$
\end{tabular} & \multicolumn{8}{|c|}{ UV-Spectrophotometric methods } & $\begin{array}{l}\text { Reported } \\
\text { method** }\end{array}$ & \multicolumn{9}{|c|}{ UV-Spectrophotometric methods } & $\begin{array}{l}\text { Reported } \\
\text { method }^{* *}\end{array}$ \\
\hline & \multicolumn{5}{|c|}{ RWD } & \multirow{2}{*}{\begin{tabular}{|c|}
${ }^{3}$ DDRD \\
\\
$\infty$ \\
\multirow{\Upsilon}{*}{}
\end{tabular}} & ${ }^{1} D$ & \multicolumn{7}{|c|}{ RWD } & \multirow{2}{*}{ 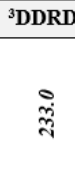 } & \multirow{3}{*}{$\begin{array}{c}\frac{\text { method }^{* *}}{{ }^{I} D} \\
\\
\\
\end{array}$} & \multicolumn{5}{|c|}{ RWD } & \multicolumn{4}{|c|}{${ }^{3}$ DDRD } & \\
\hline & 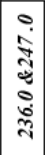 & 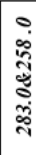 & 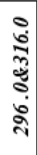 & 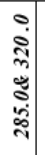 & 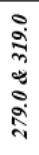 & & \multirow[t]{2}{*}{ ஜे } & 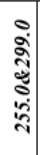 & 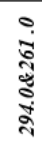 & 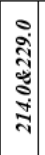 & 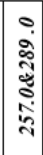 & 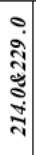 & 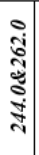 & 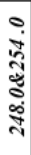 & & & 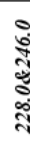 & 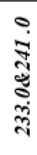 & 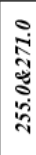 & 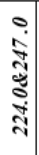 & 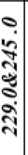 & ثं & ì & & & \\
\hline Conc. range & \multicolumn{6}{|c|}{$6.0-50.0 \mathrm{ug} \mathrm{mL}^{-1}$} & & \multicolumn{8}{|c|}{$2.0-27.0 \mathrm{ug} \mathrm{mL}^{-1}$} & & \multicolumn{8}{|c|}{$3.0-25.0 \mathrm{ug} \mathrm{mL}^{-1}$} & & \\
\hline Mean \% & ๙ิ & $\begin{array}{l}8 \\
\stackrel{8}{0} \\
\end{array}$ & $\begin{array}{l}\text { శ్ } \\
\stackrel{0}{0}\end{array}$ & $\begin{array}{l}0 \\
\vdots \\
0 \\
0\end{array}$ & $\begin{array}{l}\text { gे } \\
\text { वे. }\end{array}$ & $\begin{array}{l}\vec{\sigma} \\
\overrightarrow{0}\end{array}$ & $\begin{array}{l}\text { if } \\
8 \\
0\end{array}$ & $\mid \begin{array}{l}0 \\
0 \\
0 \\
0\end{array}$ & $\begin{array}{l}\text { m. } \\
\stackrel{0}{\circ}\end{array}$ & $\mid \begin{array}{l}0 \\
0 \\
0 \\
0\end{array}$ & กิ & $\begin{array}{l}0 \\
0 \\
\vdots \\
0\end{array}$ & 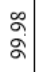 & ? & $\begin{array}{l}\text { : } \\
\stackrel{\Delta}{\alpha}\end{array}$ & ஸे & ฮิ & $\begin{array}{l}\tilde{\alpha} \\
\text { ò }\end{array}$ & 苗 & $\begin{array}{l}\text { : } \\
\vdots \\
\vdots\end{array}$ & $\begin{array}{l}\text { in } \\
\text { a }\end{array}$ & $\begin{array}{l}0 \\
\stackrel{6}{0} \\
\vdots \\
0\end{array}$ & $\begin{array}{l}\tilde{\sigma} \\
\text { }\end{array}$ & $\begin{array}{l}\overrightarrow{8} \\
\stackrel{8}{\circ}\end{array}$ & مْ & $\begin{array}{l}\stackrel{0}{0} \\
\stackrel{0}{0}\end{array}$ \\
\hline S.D. & 声 & $\stackrel{n}{\circ}$ & $\stackrel{\vec{m}}{\rightarrow}$ & ণे. & $\hat{\circ}$ & 5 & :0 & m. & $\stackrel{9}{-1}$ & $\vec{m}$ & 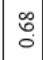 & $\vec{m}$ & $\stackrel{\circ}{-}$ & क्ष & $\stackrel{\circ}{-}$ & $\stackrel{\circ}{\circ}$ & 인 & 용 & $\hat{m}$ & $\begin{array}{l}0 \\
\vdots \\
c\end{array}$ & लี & 옹 & $\stackrel{\circ}{\leftrightarrows}$ & f. & ळ & $\stackrel{n}{\circ}$ \\
\hline Variance & 웅 & 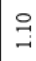 & $\stackrel{2}{-i}$ & $\stackrel{t}{\Delta}$ & 옹 & $\underset{0}{\ddagger}$ & รี & $\stackrel{\check{s}}{a}$ & $\underset{-}{F}$ & $\vec{i}$ & f & ت & $\stackrel{\infty}{\exists}$ & : & $\exists$ & లి & $\stackrel{8}{-}$ & $\begin{array}{c}0 \\
0 \\
0\end{array}$ & $\overline{0}$ & $\vec{m}$ & $\underline{z}$ & $\stackrel{\infty}{\circ}$ & $\approx$ & กิ & ¿̊. & ñ \\
\hline $\mathbf{N}$ & 5 & 5 & 5 & 5 & 5 & 5 & 5 & 5 & 5 & 5 & 5 & 5 & 5 & 5 & 5 & 5 & 5 & 5 & 5 & 5 & 5 & 5 & 5 & 5 & 5 & 5 \\
\hline t- test $^{*}$ & $\stackrel{2}{\check{c}}$ & i & $\vec{m}$ & $\begin{array}{c}8 \\
- \\
-1\end{array}$ & $\stackrel{\circ}{\circ}$ & $\stackrel{\infty}{\infty}$ & & : & $\stackrel{\text { i }}{\text { i }}$ & तิ & i & $\exists$ & $\overrightarrow{\vec{\infty}}$ & $\stackrel{-}{9}$ & $\stackrel{6}{-}$ & . & ః & $\stackrel{\infty}{\check{\perp}}$ & $\stackrel{\circ}{-}$ & $\begin{array}{l}0 \\
0 \\
0\end{array}$ & 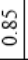 & in & : & F. & $\stackrel{\infty}{0}$ & \\
\hline$F-$ test $^{*}$ & $\stackrel{+}{+}$ & $\vec{b}$ & $\stackrel{+}{+}$ & $\stackrel{2}{-1}$ & ڤ̆ & $\stackrel{-}{\circ}$ & & $\stackrel{-5}{7}$ & ڤू & $\begin{array}{l}2 \\
+7\end{array}$ & $\vec{m}$ & $\stackrel{2}{+}$ & $\vec{m}$ & 古 & $\stackrel{n}{m}$ & ' & ะั & 옹 & $\vec{F}$ & $\begin{array}{l}\infty \\
- \\
-\end{array}$ & $\stackrel{0}{\circ}$ & $\stackrel{1}{\infty}$ & $\stackrel{\infty}{i}$ & m. & $\exists$ & \\
\hline $\begin{array}{l}\text { Standard addition } \\
\text { Mean } \% \pm \text { S.D. }\end{array}$ & 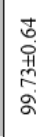 & 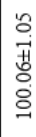 & 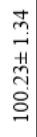 & $\begin{array}{c}0 \\
0 \\
0 \\
0 \\
+ \\
0 \\
0 \\
0 \\
0 \\
-1\end{array}$ & 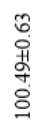 & $\begin{array}{l}\hat{0} \\
0 \\
00 \\
0 \\
-0 \\
0\end{array}$ & & 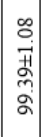 & 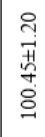 & 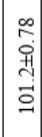 & 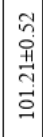 & 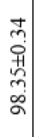 & $\begin{array}{l}0 \\
0 \\
0 \\
\vdots \\
0 \\
\infty \\
\vdots\end{array}$ & 官 & 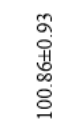 & ' & $\begin{array}{l}\text { ㅇ } \\
\text { 풍 } \\
\text { 응 }\end{array}$ & 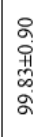 & $\begin{array}{c}m \\
\hat{o} \\
i \\
m \\
o \\
o \\
0\end{array}$ & 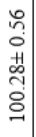 & लै & $\begin{array}{l}\stackrel{0}{0} \\
0 \\
110 \\
0 \\
0 \\
0\end{array}$ & 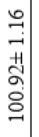 & 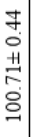 & 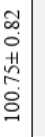 & \\
\hline
\end{tabular}

*Figures in parenthesis are the theoretical $\mathrm{t}$ - and $\mathrm{F}$ - values at $\mathrm{p}=0.05$ were 2.31 and 6.39 ; respectively. **The reported method involved UVmeasurements for ${ }^{1} \mathrm{D}$ of both amprolium and ethopabate determination ${ }^{20}$, TLC densitometry for determination of sulfaquinoxaline-Na ${ }^{18}$ 


\section{CONCLUSION}

The present work provides two simple, rapid, accurate, precise, time and cost reduction methods. Both methods could be used for the simultaneous routine analysis of amprolium $\mathrm{HCl}$, sulfaquinoxaline-

$\mathrm{Na}$ and ethopabate in their pure and available dosage forms without preliminarily sample separation and preparation. The proposed UVspectrophotometric methods were more selective. Because they are used for determination of the three drugs in presence of each other without any interference and do not need any expansive apparatus. Therefore, they could be applied in quality control laboratories for analysis of cited drugs.

\section{Conflict of interest} Ethics.

The authors declare no conflict of interest

\section{Ethics statement: NA}

\section{Author contribution}

Author SA designed the study and wrote the protocol. Author NS supervise the analyses of the study. Author SE performed the experimental work, statistical analysis and wrote the first draft of the manuscript and managed literature searches. All authors read and approved the final manuscript.

\section{Funding}

The work received no funding.

\section{REFERENCES}

1. Chapman HD, Barta JR, Black D, Gruber A, Jenkins M, Nicholas CS, et. al. A selective review of advances in coccidiosis research. Adv. in parasitol. 2013 Jan 01; 83: 93-171.

2. The United States Pharmacopeia 43, NF 38, Asian Ed., Rand Mc Nally, USA, 2020.

3. Duszynski D, Kvičerová J and Seville R S. The Biology and Identification of the Coccidia (Apicomplexa) of Carnivores of the World, Chapter 18 - Treatment and Drug Therapies of Coccidiosis in Carnivora.2018 Jun; 445-463.

4. Peek HW and Landman WJM. Coccidiosis in poultry: anticoccidial products, vaccines and other prevention strategies. Vet. Q. 2011 Aug 23; 31(3): 143-161.
5. Lakkanatinaporn P. and Matayatsuk C. Simultaneous HPLC method for determination of sodium trimethoprim phenyl-propanol disulphonate and sodium sulfaquinoxaline in veterinary drugs. Songklanakarin J. Sci. Technol. 2004 Nov; 26(6): 849-854.

6. Ali MM, Algozoly MA and Shinger MI. Development and validation of RP-HPLC method for simultaneous determination of amprolium HCL and ethopabate in their combination drug. Chemical and Biomolecular Engineering. 2017 Feb 24; 2(1): 51-56.

7. Ghanem M, Abu-Lafi S and Mohammad D. Development and validation of a stability indicating hydrephilic interaction liquid chromatographic method for the determination of sulfaquinoxaline sodium in water soluble powder formulation. International Journal of Pharmacy and Pharmaceutical Sciences. 2014 Jan 09; 6(2): 652-657.

8. Bakr MM, El-Kafrawy DS, Abdel-Khalek MM and Belal TS. Comprehensive stabilityindicating high-performance liquid chromatography coupled with diode array detection method for simultaneous determination of amprolium hydrochloride and ethopabate in powder dosage form for veterinary use. J. Sep. Sc.2019 Sep 11; 42(21): 3340-3351.

9. Ghanem M, Abu-Lafi S, Karaman R and Hallak H. Validated HPLC method to simultaneously determine amprolium hydrochloride, sulfaquinoxaline sodium and vitamin $\mathrm{k} 3$ in a.s.k powder on ZIC-HILIC column. Pharmaceut. Anal. Acta. 2012 Sep 25; 3(7): 168.

10. Furusawa N. Simplified high-performance liquid chromatographic determination of residual amprolium in edible chicken tissues. J. Chromatogr. Sci. 2002 Aug 01; 40(7): 355358.

11. Kim B, Hyunsun H, Joo LJ, Yong CN and Woon MS. Determination of coccidiostats (amprolium and decoquinate) in cattle and chicken's muscle using high performance liquid chromatography. Bull. Korean Chem. Soc. 2012 Dec 13; 33(2): 559-563. 
12. Mantri AP, Rubeena MS, Kandepu N and Kumar S. Simultaneous estimation of sulfaquinoxaline sodium and amprolium hydrochloride by RP- HPLC. Int. J. Pharm. Sci. 2015 Mar 01; 6(3): 1097-1100.

13. Song W, Huang M, Rumbeiha $\mathrm{W}$ and Li $\mathrm{H}$. Determination of amprolium, carbadox, monensin, and tylosin in surface water by liquid chromatography/tandem mass spectrometry. Rapid Commun. Mass Spectrom. 2007 May 17; 21(12): 1944-1950

14. Stefania S, Mauro C, Gian F, Giuseppina A and Maria A. Determination of amprolium in feed by a liquid chromatography-mass spectrometry method. J. Pharm. Biomed. 2008 Dec 15; 48(5): 1457-1461.

15. Liu R, He P, Li Z and Li R. Simultaneous determination of 16 sulfonamides in animal feeds by UHPLC-MS-MS. J. Chromatogr Sci. 2011 Sep 01; 49(8):640-646.

16. El-Kosasy AM, Lobna AH, Magdy $\mathrm{N}$ and Mahmoud MA. Validated TLC-densitometric method for determination of amprolium hydrochloride and ethopabate in veterinary preparation. Analytical Chemistry: An Indian Journal. 2016 Jun; 16(13) 1-11.

17. Basha M, El-Rahman M, BebawyL and Moustafa, A. Validated TLC stability indicating methods for the quantitative determination of some veterinary drugs. Microchem. J. 2018 May; 146: 157-163.

18. Razeq S, Demerdash A, Fouad M and Sanabary H. Densitometric and ratio spectra methods for simultaneous determination of sulfaquinoxaline sodium and pyrimethamine in binary mixture. Bull. Fac. Pharm. Cairo Univ. 2019 Jun 09; 57 (1): $35-45$.

19. El-Kosasy A, Hussein L, Magdy N and Mahmoud MA. Sensitive spectrofluorimetric methods for determination of ethopabate and amprolium hydrochloride in chicken plasma and their residues in food samples. Spectrochim. Acta A Mol. Biomol. Spectrosc. 2015 Nov 5; 150: 430-439.

20. Alomary A. Simultaneous Quantification of amprolium and ethopabate in pharmaceutical preparation by derivative ultraviolet spectroscopy. Abhath Al-Yarmouk Pure
Science and Engineering Series. 2004; 13(1): 59-70.

21. Soleymanpour A and Rezvani SA. Development of a novel carbon paste sensor for determination of micromolar amounts of sulfaquinoxaline in pharmaceutical and biological samples. Mater. Sci. Eng. C. 2016 Jan 01; 58: 504-509.

22. Basha MA, Abd El-Rahman M, Bebawy LI and Salem MY. Novel potentiometric application for the determination of amprolium $\mathrm{HCl}$ in its single and combined dosage form and in chicken liver. Chin. Chem. Lett. 2017 Mar; 28(3): 612-618.

23. Hajian $R$ and Afshari N. The spectrophotometric multicomponent analysis of a ternary mixture of ibuprofen, caffeine and paracetamol by the combination of double divisor-ratio spectra derivative and h-point standard addition method. E- J. Chem. 2012 Jan 15; 9(3): 1153-1164.

24. Kamal AH, El-Malla SF and Hammad SF. A review on UV spectrophotometric methods for simultaneous multicomponent analysis. Eur. J. Pharm. Sci. 2016 Jan 27; 3(2): 348-360.

25. Saad AS. Novel Spectrophotometric method for selective determination of compounds in ternary mixtures (dual wavelength in ratio spectra). Spectrochim. Acta A Mol. Biomol. Spectrosc. 2015 Aug 5; 147: 257-261.

26. The International Conference on Harmonization. Q2 (R1), Validation of Analytical Procedure Text and Methodology, Geneva, 2005.

27. Harris D.C.; Quantitative Chemical Analysis, 8th Ed., W.H. Freeman and Company, USA, Chap. 4 and 18, 2010. 